\title{
Some properties of the generalized Apostol-type polynomials
}

Da-Qian Lu' and Qiu-Ming Luo ${ }^{2 *}$

\section{"Correspondence:}

luomath2007@163.com

${ }^{2}$ Department of Mathematics,

Chongqing Normal University,

Chongqing Higher Education Mega

Center, Huxi Campus, Chongqing,

401331, People's Republic of China

Full list of author information is

available at the end of the article

\begin{abstract}
In this paper, we study some properties of the generalized Apostol-type polynomials (see (Luo and Srivastava in Appl. Math. Comput. 217:5702-5728, 2011)), including the recurrence relations, the differential equations and some other connected problems, which extend some known results. We also deduce some properties of the generalized Apostol-Euler polynomials, the generalized Apostol-Bernoulli polynomials, and Apostol-Genocchi polynomials of high order.
\end{abstract}

MSC: Primary 11B68; secondary 33C65

Keywords: generalized Apostol type polynomials; recurrence relations; differential equations; connected problems; quasi-monomial

\section{Introduction, definitions and motivation}

The classical Bernoulli polynomials $B_{n}(x)$, the classical Euler polynomials $E_{n}(x)$ and the classical Genocchi polynomials $G_{n}(x)$, together with their familiar generalizations $B_{n}^{(\alpha)}(x)$, $E_{n}^{(\alpha)}(x)$ and $G_{n}^{(\alpha)}(x)$ of (real or complex) order $\alpha$, are usually defined by means of the following generating functions (see, for details, [1], pp.532-533 and [2], p.61 et seq.; see also [3] and the references cited therein):

$$
\begin{aligned}
& \left(\frac{z}{e^{z}-1}\right)^{\alpha} \cdot e^{x z}=\sum_{n=0}^{\infty} B_{n}^{(\alpha)}(x) \frac{z^{n}}{n !} \quad(|z|<2 \pi), \\
& \left(\frac{2}{e^{z}+1}\right)^{\alpha} \cdot e^{x z}=\sum_{n=0}^{\infty} E_{n}^{(\alpha)}(x) \frac{z^{n}}{n !} \quad(|z|<\pi)
\end{aligned}
$$

and

$$
\left(\frac{2 z}{e^{z}+1}\right)^{\alpha} \cdot e^{x z}=\sum_{n=0}^{\infty} G_{n}^{(\alpha)}(x) \frac{z^{n}}{n !} \quad(|z|<\pi)
$$

So that, obviously, the classical Bernoulli polynomials $B_{n}(x)$, the classical Euler polynomials $E_{n}(x)$ and the classical Genocchi polynomials $G_{n}(x)$ are given, respectively, by

$$
B_{n}(x):=B_{n}^{(1)}(x), \quad E_{n}(x):=E_{n}^{(1)}(x) \quad \text { and } \quad G_{n}(x):=G_{n}^{(1)}(x) \quad\left(n \in \mathbb{N}_{0}\right)
$$


For the classical Bernoulli numbers $B_{n}$, the classical Euler numbers $E_{n}$ and the classical Genocchi numbers $G_{n}$ of order $n$, we have

$$
B_{n}:=B_{n}(0)=B_{n}^{(1)}(0), \quad E_{n}:=E_{n}(0)=E_{n}^{(1)}(0) \quad \text { and } \quad G_{n}:=G_{n}(0)=G_{n}^{(1)}(0) \text {, }
$$

respectively.

Some interesting analogues of the classical Bernoulli polynomials and numbers were first investigated by Apostol (see [4], p.165, Eq. (3.1)) and (more recently) by Srivastava (see [5], pp.83-84). We begin by recalling here Apostol's definitions as follows.

Definition 1.1 (Apostol [4]; see also Srivastava [5]) The Apostol-Bernoulli polynomials $\mathcal{B}_{n}(x ; \lambda)(\lambda \in \mathbb{C})$ are defined by means of the following generating function:

$$
\begin{aligned}
& \frac{z e^{x z}}{\lambda e^{z}-1}=\sum_{n=0}^{\infty} \mathcal{B}_{n}(x ; \lambda) \frac{z^{n}}{n !} \\
& \quad(|z|<2 \pi \text { when } \lambda=1 ;|z|<|\log \lambda| \text { when } \lambda \neq 1)
\end{aligned}
$$

with, of course,

$$
B_{n}(x)=\mathcal{B}_{n}(x ; 1) \quad \text { and } \quad \mathcal{B}_{n}(\lambda):=\mathcal{B}_{n}(0 ; \lambda)
$$

where $\mathcal{B}_{n}(\lambda)$ denotes the so-called Apostol-Bernoulli numbers.

Recently, Luo and Srivastava [6] further extended the Apostol-Bernoulli polynomials as the so-called Apostol-Bernoulli polynomials of order $\alpha$.

Definition 1.2 (Luo and Srivastava [6]) The Apostol-Bernoulli polynomials $\mathcal{B}_{n}^{(\alpha)}(x ; \lambda)(\lambda \in$ $\mathbb{C}$ ) of order $\alpha \in \mathbb{N}_{0}$ are defined by means of the following generating function:

$$
\begin{aligned}
& \left(\frac{z}{\lambda e^{z}-1}\right)^{\alpha} \cdot e^{x z}=\sum_{n=0}^{\infty} \mathcal{B}_{n}^{(\alpha)}(x ; \lambda) \frac{z^{n}}{n !} \\
& \quad(|z|<2 \pi \text { when } \lambda=1 ;|z|<|\log \lambda| \text { when } \lambda \neq 1)
\end{aligned}
$$

with, of course,

$$
B_{n}^{(\alpha)}(x)=\mathcal{B}_{n}^{(\alpha)}(x ; 1) \quad \text { and } \quad \mathcal{B}_{n}^{(\alpha)}(\lambda):=\mathcal{B}_{n}^{(\alpha)}(0 ; \lambda)
$$

where $\mathcal{B}_{n}^{(\alpha)}(\lambda)$ denotes the so-called Apostol-Bernoulli numbers of order $\alpha$.

On the other hand, Luo [7], gave an analogous extension of the generalized Euler polynomials as the so-called Apostol-Euler polynomials of order $\alpha$.

Definition 1.3 (Luo [7]) The Apostol-Euler polynomials $\mathcal{E}_{n}^{(\alpha)}(x ; \lambda)(\lambda \in \mathbb{C})$ of order $\alpha \in \mathbb{N}_{0}$ are defined by means of the following generating function:

$$
\left(\frac{2}{\lambda e^{z}+1}\right)^{\alpha} \cdot e^{x z}=\sum_{n=0}^{\infty} \mathcal{E}_{n}^{(\alpha)}(x ; \lambda) \frac{z^{n}}{n !} \quad(|z|<|\log (-\lambda)|)
$$


with, of course,

$$
E_{n}^{(\alpha)}(x)=\mathcal{E}_{n}^{(\alpha)}(x ; 1) \quad \text { and } \quad \mathcal{E}_{n}^{(\alpha)}(\lambda):=\mathcal{E}_{n}^{(\alpha)}(0 ; \lambda)
$$

where $\mathcal{E}_{n}^{(\alpha)}(\lambda)$ denotes the so-called Apostol-Euler numbers of order $\alpha$.

On the subject of the Genocchi polynomials $G_{n}(x)$ and their various extensions, a remarkably large number of investigations have appeared in the literature (see, for example, [8-14]). Moreover, Luo (see [12-14]) introduced and investigated the Apostol-Genocchi polynomials of (real or complex) order $\alpha$, which are defined as follows:

Definition 1.4 The Apostol-Genocchi polynomials $\mathcal{G}_{n}^{(\alpha)}(x ; \lambda)(\lambda \in \mathbb{C})$ of order $\alpha \in \mathbb{N}_{0}$ are defined by means of the following generating function:

$$
\left(\frac{2 z}{\lambda e^{z}+1}\right)^{\alpha} \cdot e^{x z}=\sum_{n=0}^{\infty} \mathcal{G}_{n}^{(\alpha)}(x ; \lambda) \frac{z^{n}}{n !} \quad(|z|<|\log (-\lambda)|)
$$

with, of course,

$$
\begin{aligned}
& G_{n}^{(\alpha)}(x)=\mathcal{G}_{n}^{(\alpha)}(x ; 1), \quad \mathcal{G}_{n}^{(\alpha)}(\lambda):=\mathcal{G}_{n}^{(\alpha)}(0 ; \lambda), \\
& \mathcal{G}_{n}(x ; \lambda):=\mathcal{G}_{n}^{(1)}(x ; \lambda) \quad \text { and } \quad \mathcal{G}_{n}(\lambda):=\mathcal{G}_{n}^{(1)}(\lambda),
\end{aligned}
$$

where $\mathcal{G}_{n}(\lambda), \mathcal{G}_{n}^{(\alpha)}(\lambda)$ and $\mathcal{G}_{n}(x ; \lambda)$ denote the so-called Apostol-Genocchi numbers, the Apostol-Genocchi numbers of order $\alpha$ and the Apostol-Genocchi polynomials, respectively.

Recently, Luo and Srivastava [15] introduced a unification (and generalization) of the above-mentioned three families of the generalized Apostol type polynomials.

Definition 1.5 (Luo and Srivastava [15]) The generalized Apostol type polynomials $\mathcal{F}_{n}^{(\alpha)}(x ; \lambda ; u, v)\left(\alpha \in \mathbb{N}_{0}, \lambda, u, v \in \mathbb{C}\right)$ of order $\alpha$ are defined by means of the following generating function:

$$
\left(\frac{2^{u} z^{v}}{\lambda e^{z}+1}\right)^{\alpha} \cdot e^{x z}=\sum_{n=0}^{\infty} \mathcal{F}_{n}^{(\alpha)}(x ; \lambda ; u, v) \frac{z^{n}}{n !} \quad(|z|<|\log (-\lambda)|)
$$

where

$$
\mathcal{F}_{n}^{(\alpha)}(\lambda ; u, v):=\mathcal{F}_{n}^{(\alpha)}(0 ; \lambda ; u, v)
$$

denote the so-called Apostol type numbers of order $\alpha$.

So that, by comparing Definition 1.5 with Definitions 1.2,1.3 and 1.4, we have

$$
\begin{aligned}
& \mathcal{B}_{n}^{(\alpha)}(x ; \lambda)=(-1)^{\alpha} \mathcal{F}_{n}^{(\alpha)}(x ;-\lambda ; 0,1), \\
& \mathcal{E}_{n}^{(\alpha)}(x ; \lambda)=\mathcal{F}_{n}^{(\alpha)}(x ; \lambda ; 1,0), \\
& \mathcal{G}_{n}^{(\alpha)}(x ; \lambda)=\mathcal{F}_{n}^{(\alpha)}(x ; \lambda ; 1,1) .
\end{aligned}
$$


A polynomial $p_{n}(x)(n \in \mathbb{N}, x \in \mathbb{C})$ is said to be a quasi-monomial [16], whenever two operators $\hat{M}, \hat{P}$, called multiplicative and derivative (or lowering) operators respectively, can be defined in such a way that

$$
\begin{aligned}
& \hat{P} p_{n}(x)=n p_{n-1}(x), \\
& \hat{M} p_{n}(x)=p_{n+1}(x),
\end{aligned}
$$

which can be combined to get the identity

$$
\hat{M} \hat{P} p_{n}(x)=n p_{n}(x)
$$

The Appell polynomials [17] can be defined by considering the following generating function:

$$
A(t) e^{x t}=\sum_{n=0}^{\infty} \frac{R_{n}(x)}{n !} t^{n}
$$

where

$$
A(t)=\sum_{k=0}^{\infty} \frac{R_{k}}{k !} t^{k} \quad(A(0) \neq 0)
$$

is analytic function at $t=0$.

From [18], we know that the multiplicative and derivative operators of $R_{n}(x)$ are

$$
\begin{aligned}
& \hat{M}=\left(x+\alpha_{0}\right)+\sum_{k=0}^{n-1} \frac{\alpha_{n-k}}{(n-k) !} D_{x}^{n-k}, \\
& \hat{P}=D_{x},
\end{aligned}
$$

where

$$
\frac{A^{\prime}(t)}{A(t)}=\sum_{n=0}^{\infty} \alpha_{n} \frac{t^{n}}{n !}
$$

By using (1.21), we have the following lemma.

Lemma 1.6 ([18]) The Appell polynomials $R_{n}(x)$ defined by (1.22) satisfy the differential equation:

$$
\frac{\alpha_{n-1}}{(n-1) !} y^{(n)}+\frac{\alpha_{n-2}}{(n-2) !} y^{(n-1)}+\cdots+\frac{\alpha_{1}}{1 !} y^{\prime \prime}+\left(x+\alpha_{0}\right) y^{\prime}-n y=0
$$

where the numerical coefficients $\alpha_{k}, k=1,2, \ldots, n-1$ are defined in (1.26), and are linked to the values $R_{k}$ by the following relations:

$$
R_{k+1}=\sum_{h=0}^{k}\left(\begin{array}{l}
k \\
h
\end{array}\right) R_{h} \alpha_{k-h} .
$$


Let $\mathcal{P}$ be the vector space of polynomials with coefficients in $\mathbb{C}$. A polynomial sequence $\left\{P_{n}\right\}_{n \geq 0}$ be a polynomial set. $\left\{P_{n}\right\}_{n \geq 0}$ is called a $\sigma$-Appell polynomial set of transfer power series $A$ is generated by

$$
G(x, t)=A(t) G_{0}(x, t)=\sum_{n=0}^{\infty} \frac{P_{n}(x)}{n !} t^{n}
$$

where $G_{0}(x, t)$ is a solution of the system:

$$
\begin{aligned}
& \sigma G_{0}(x, t)=t G_{0}(x, t), \\
& G_{0}(x, 0)=1 .
\end{aligned}
$$

In [19], the authors investigated the connection coefficients between two polynomials. And there is a result about connection coefficients between two $\sigma$-Appell polynomial sets.

Lemma 1.7 ([19]) Let $\sigma \in \Lambda^{(-1)}$. Let $\left\{P_{n}\right\}_{n \geq 0}$ and $\left\{Q_{n}\right\}_{n \geq 0}$ be two $\sigma$-Appell polynomial sets of transfer power series, respectively, $A_{1}$ and $A_{2}$. Then

$$
Q_{n}(x)=\sum_{m=0}^{n} \frac{n !}{m !} \alpha_{n-m} P_{m}(x)
$$

where

$$
\frac{A_{2}(t)}{A_{1}(t)}=\sum_{k=0}^{\infty} \alpha_{k} t^{k}
$$

In recent years, several authors obtained many interesting results involving the related Bernoulli polynomials and Euler polynomials [5, 20-40]. And in [29], the authors studied some series identities involving the generalized Apostol type and related polynomials.

In this paper, we study some other properties of the generalized Apostol type polynomials $\mathcal{F}_{n}^{(\alpha)}(x ; \lambda ; u, v)$, including the recurrence relations, the differential equations and some connection problems, which extend some known results. As special, we obtain some properties of the generalized Apostol-Euler polynomials, the generalized Apostol-Bernoulli polynomials and Apostol-Genocchi polynomials of high order.

\section{Recursion formulas and differential equations}

From the generating function (1.14), we have

$$
\frac{\partial}{\partial x} \mathcal{F}_{n}^{(\alpha)}(x ; \lambda ; u, v)=n \mathcal{F}_{n-1}^{(\alpha)}(x ; \lambda ; u, v) .
$$

A recurrence relation for the generalized Apostol type polynomials is given by the following theorem.

Theorem 2.1 For any integral $n \geq 1, \lambda \in \mathbb{C}$ and $\alpha \in \mathbb{N}$, the following recurrence relation for the generalized Apostol type polynomials $\mathcal{F}_{n}^{(\alpha)}(x ; \lambda ; u, v)$ holds true:

$$
\left(\frac{\alpha v}{n+1}-1\right) \mathcal{F}_{n+1}^{(\alpha)}(x ; \lambda ; u, v)=\frac{\alpha \lambda}{2^{u}} \cdot \frac{n !}{(n+v) !} \mathcal{F}_{n+v}^{(\alpha+1)}(x+1 ; \lambda ; u, v)-x \mathcal{F}_{n}^{(\alpha)}(x ; \lambda ; u, v) .
$$


Proof Differentiating both sides of (1.14) with respect to $t$, and using some elementary algebra and the identity principle of power series, recursion (2.2) easily follows.

By setting $\lambda:=-\lambda, u=0$ and $v=1$ in Theorem 2.1 , and then multiplying $(-1)^{\alpha}$ on both sides of the result, we have:

Corollary 2.2 For any integral $n \geq 1, \lambda \in \mathbb{C}$ and $\alpha \in \mathbb{N}$, the following recurrence relation for the generalized Apostol-Bernoulli polynomials $\mathcal{B}_{n}^{(\alpha)}(x ; \lambda)$ holds true:

$$
[\alpha-(n+1)] \mathcal{B}_{n+1}^{(\alpha)}(x ; \lambda)=\alpha \lambda \mathcal{B}_{n+1}^{(\alpha+1)}(x+1 ; \lambda)-x \mathcal{B}_{n}^{(\alpha)}(x ; \lambda) .
$$

By setting $u=1$ and $v=0$ in Theorem 2.1, we have the following corollary.

Corollary 2.3 For any integral $n \geq 1, \lambda \in \mathbb{C}$ and $\alpha \in \mathbb{N}$, the following recurrence relation for the generalized Apostol-Euler polynomials $\mathcal{E}_{n}^{(\alpha)}(x ; \lambda)$ holds true:

$$
\mathcal{E}_{n+1}^{(\alpha)}(x ; \lambda)=x \mathcal{E}_{n}^{(\alpha)}(x ; \lambda)-\frac{\alpha \lambda}{2} \mathcal{E}_{n}^{(\alpha+1)}(x+1 ; \lambda) .
$$

By setting $u=1$ and $v=1$ in Theorem 2.1, we have the following corollary.

Corollary 2.4 For any integral $n \geq 1, \lambda \in \mathbb{C}$ and $\alpha \in \mathbb{N}$, the following recurrence relation for the generalized Apostol-Genocchi polynomials $\mathcal{G}_{n}^{(\alpha)}(x ; \lambda)$ holds true:

$$
2[\alpha-(n+1)] \mathcal{G}_{n+1}^{(\alpha)}(x ; \lambda)=\alpha \lambda \mathcal{G}_{n+1}^{(\alpha+1)}(x+1 ; \lambda)-2(n+1) x \mathcal{G}_{n}^{(\alpha)}(x ; \lambda)
$$

From (1.14) and (1.22), we know that the generalized Appostol type polynomials $\mathcal{F}_{n}^{(\alpha)}(x ; \lambda ; u, v)$ is Appell polynomials with

$$
A(t)=\left(\frac{2^{u} t^{v}}{\lambda e^{t}+1}\right)^{\alpha}
$$

From the Eq. (23) of [15], we know that $\mathcal{G}_{0}(1 ; \lambda)=0$. So from (2.6) and (1.12), we can obtain that if $v=0$, we have

$$
\frac{A^{\prime}(t)}{A(t)}=\frac{\lambda \alpha}{2} \sum_{n=0}^{\infty} \frac{\mathcal{G}_{n+1}(1 ; \lambda)}{n+1} \cdot \frac{t^{n}}{n !} .
$$

By using (1.24) and (1.26), we can obtain the multiplicative and derivative operators of the generalized Appostol type polynomials $\mathcal{F}_{n}^{(\alpha)}(x ; \lambda ; u, v)$

$$
\begin{aligned}
& \hat{M}=\left(x+\frac{\lambda \alpha}{2} \mathcal{G}_{1}(1 ; \lambda)\right)+\frac{\lambda \alpha}{2} \sum_{k=0}^{n-1} \frac{\mathcal{G}_{n-k+1}(1 ; \lambda)}{(n-k+1) !} D_{x}^{n-k}, \\
& \hat{P}=D_{x} .
\end{aligned}
$$

From (2.1), we can obtain

$$
\frac{\partial^{p}}{\partial x^{p}} \mathcal{F}_{n}^{(\alpha)}(x ; \lambda ; u, v)=\frac{n !}{(n-p) !} \mathcal{F}_{n-p}^{(\alpha)}(x ; \lambda ; u, v) .
$$

Then by using (1.20), (2.8) and (2.10), we obtain the following result. 
Theorem 2.5 For any integral $n \geq 1, \lambda \in \mathbb{C}$ and $\alpha \in \mathbb{N}$, the following recurrence relation for the generalized Apostol type polynomials $\mathcal{F}_{n}^{(\alpha)}(x ; \lambda ; u, 0)$ holds true:

$$
\begin{aligned}
\mathcal{F}_{n+1}^{(\alpha)}(x ; \lambda ; u, 0)= & \left(x+\frac{\lambda \alpha}{2} \mathcal{G}_{1}(1 ; \lambda)\right) \mathcal{F}_{n}^{(\alpha)}(x ; \lambda ; u, 0) \\
& +\frac{\lambda \alpha}{2} \sum_{k=0}^{n-1}\left(\begin{array}{l}
n \\
k
\end{array}\right) \frac{\mathcal{G}_{n-k+1}(1 ; \lambda)}{n-k+1} \mathcal{F}_{n-k}^{(\alpha)}(x ; \lambda ; u, 0) .
\end{aligned}
$$

By setting $u=1$ in Theorem 2.5 , we have the following corollary.

Corollary 2.6 For any integral $n \geq 1, \lambda \in \mathbb{C}$ and $\alpha \in \mathbb{N}$, the following recurrence relation for the generalized Apostol-Euler polynomials $\mathcal{E}_{n}^{(\alpha)}(x ; \lambda)$ holds true:

$$
\mathcal{E}_{n+1}^{(\alpha)}(x ; \lambda)=\left(x+\frac{\lambda \alpha}{2} \mathcal{G}_{1}(1 ; \lambda)\right) \mathcal{E}_{n}^{(\alpha)}(x ; \lambda)+\frac{\lambda \alpha}{2} \sum_{k=0}^{n-1}\left(\begin{array}{l}
n \\
k
\end{array}\right) \frac{\mathcal{G}_{n-k+1}(1 ; \lambda)}{n-k+1} \mathcal{E}_{n-k}^{(\alpha)}(x ; \lambda) .
$$

Furthermore, applying Lemma 1.7 to $\mathcal{F}_{n}^{(\alpha)}(x ; \lambda ; u, 0)$, we have the following theorem.

Theorem 2.7 The generalized Apostol type polynomials $\mathcal{F}_{n}^{(\alpha)}(x ; \lambda ; u, 0)$ satisfy the differential equation:

$$
\begin{aligned}
\frac{\lambda \alpha}{2} & \frac{\mathcal{G}_{n}(1 ; \lambda)}{n !} y^{(n)}+\frac{\lambda \alpha}{2} \frac{\mathcal{G}_{n-1}(1 ; \lambda)}{(n-1) !} y^{(n-1)}+\cdots \\
& +\frac{\lambda \alpha}{2} \frac{\mathcal{G}_{2}(1 ; \lambda)}{2} y^{\prime \prime}+\left(x+\frac{\lambda \alpha}{2} \mathcal{G}_{1}(1 ; \lambda)\right) y^{\prime}-n y=0 .
\end{aligned}
$$

Specially, by setting $u=1$ in Theorem 2.7 , then we have the following corollary.

Corollary 2.8 The generalized Apostol-Euler polynomials $\mathcal{E}_{n}^{(\alpha)}(x ; \lambda)$ satisfy the differential equation:

$$
\begin{aligned}
\frac{\lambda \alpha}{2} & \frac{\mathcal{G}_{n}(1 ; \lambda)}{n !} y^{(n)}+\frac{\lambda \alpha}{2} \frac{\mathcal{G}_{n-1}(1 ; \lambda)}{(n-1) !} y^{(n-1)}+\cdots \\
& +\frac{\lambda \alpha}{2} \frac{\mathcal{G}_{2}(1 ; \lambda)}{2} y^{\prime \prime}+\left(x+\frac{\lambda \alpha}{2} \mathcal{G}_{1}(1 ; \lambda)\right) y^{\prime}-n y=0 .
\end{aligned}
$$

\section{Connection problems}

From (1.14) and (1.28), we know that the generalized Apostol type polynomials $\mathcal{F}_{n}^{(\alpha)}(x ; \lambda$; $u, v)$ are a $D_{x}$-Appell polynomial set, where $D_{x}$ denotes the derivative operator.

From Table 1 in [19], we know that the derivative operators of monomials $x^{n}$ and the Gould-Hopper polynomials $g_{n}^{m}(x, h)[30]$ are all $D_{x}$. And their transfer power series $A(t)$ are 1 and $e^{h t^{m}}$, respectively.

Applying Lemma 1.7 to $P_{n}(x)=x^{n}$ and $Q_{n}(x)=\mathcal{F}_{n}^{(\alpha)}(x ; \lambda ; u, v)$, we have the following theorem. 


\section{Theorem 3.1}

$$
\mathcal{F}_{n}^{(\alpha)}(x ; \lambda ; u, v)=\sum_{m=0}^{n}\left(\begin{array}{c}
n \\
m
\end{array}\right) \mathcal{F}_{n-m}^{(\alpha)}(\lambda ; u, v) x^{m}
$$

where $\mathcal{F}_{n}^{(\alpha)}(\lambda ; u, v)$ is the so-called Apostol type numbers of order $\alpha$ defined by (1.15).

By setting $\lambda:=-\lambda, u=0$ and $v=1$ in Theorem 3.1 , and then multiplying $(-1)^{\alpha}$ on both sides of the result, we have the following corollary.

\section{Corollary 3.2}

$$
\mathcal{B}_{n}^{(\alpha)}(x ; \lambda)=\sum_{m=0}^{n}\left(\begin{array}{l}
n \\
m
\end{array}\right) \mathcal{B}_{n-m}^{(\alpha)}(\lambda) x^{m}
$$

which is just Eq. (3.1) of [23].

By setting $u=0$ and $v=0$ in Theorem 3.1, we have the following corollary.

\section{Corollary 3.3}

$$
\mathcal{E}_{n}^{(\alpha)}(x ; \lambda)=\sum_{m=0}^{n}\left(\begin{array}{c}
n \\
m
\end{array}\right) \mathcal{E}_{n-m}^{(\alpha)}(\lambda) x^{m}
$$

By setting $u=1$ and $v=1$ in Theorem 3.1, we have the following corollary.

\section{Corollary 3.4}

$$
\mathcal{G}_{n}^{(\alpha)}(x ; \lambda)=\sum_{m=0}^{n}\left(\begin{array}{c}
n \\
m
\end{array}\right) \mathcal{G}_{n-m}^{(\alpha)}(\lambda) x^{m}
$$

which is just Eq. (24) of [15].

Applying Lemma 1.7 to $P_{n}(x)=\mathcal{F}_{n}(x ; \lambda ; u, v)$ and $Q_{n}(x)=\mathcal{F}_{n}^{(\alpha)}(x ; \lambda ; u, v)$, we have the following theorem.

\section{Theorem 3.5}

$$
\mathcal{F}_{n}^{(\alpha)}(x ; \lambda ; u, v)=\sum_{m=0}^{n}\left(\begin{array}{c}
n \\
m
\end{array}\right) \mathcal{F}_{n-m}^{(\alpha-1)}(\lambda ; u, v) \mathcal{F}_{m}(x ; \lambda ; u, v)
$$

where $\mathcal{F}_{n}^{(\alpha)}(\lambda ; u, v)$ is the so-called Apostol type numbers of order $\alpha$ defined by (1.15).

By setting $\lambda:=-\lambda, u=0$ and $v=1$ in Theorem 3.5 , and then multiplying $(-1)^{\alpha}$ on both sides of the result, we have the following corollary.

\section{Corollary 3.6}

$$
\mathcal{B}_{n}^{(\alpha)}(x ; \lambda)=\sum_{m=0}^{n}\left(\begin{array}{c}
n \\
m
\end{array}\right) \mathcal{B}_{n-m}^{(\alpha-1)}(\lambda) \mathcal{B}_{m}(x ; \lambda)
$$

which is just Eq. (3.2) of [23]. 
By setting $u=1$ and $v=0$ in Theorem 3.5 , we have the following corollary.

\section{Corollary 3.7}

$$
\mathcal{E}_{n}^{(\alpha)}(x ; \lambda)=\sum_{m=0}^{n}\left(\begin{array}{c}
n \\
m
\end{array}\right) \mathcal{E}_{n-m}^{(\alpha-1)}(\lambda) \mathcal{E}_{m}(x ; \lambda)
$$

By setting $u=1$ and $v=1$ in Theorem 3.5 , we have the following corollary.

\section{Corollary 3.8}

$$
\mathcal{G}_{n}^{(\alpha)}(x ; \lambda)=\sum_{m=0}^{n}\left(\begin{array}{c}
n \\
m
\end{array}\right) \mathcal{G}_{n-m}^{(\alpha-1)}(\lambda) \mathcal{G}_{m}(x ; \lambda)
$$

Applying Lemma 1.7 to $P_{n}(x)=g_{n}^{m}(x, h)$ and $Q_{n}(x)=\mathcal{F}_{n}^{(\alpha)}(x ; \lambda ; u, v)$, we have the following theorem.

\section{Theorem 3.9}

$$
\mathcal{F}_{n}^{(\alpha)}(x ; \lambda ; u, v)=\sum_{r=0}^{n} \frac{n !}{r !}\left[\sum_{k=0}^{[(n-r) / m]}(-1)^{k} \frac{h^{k}}{k !(n-r-m k) !} \mathcal{F}_{n-r-m k}^{(\alpha)}(\lambda ; u, v)\right] g_{r}^{m}(x, h) .
$$

By setting $\lambda:=-\lambda, u=0$ and $v=1$ in Theorem 3.9 , and then multiplying $(-1)^{\alpha}$ on both sides of the result, we have the following corollary.

\section{Corollary 3.10}

$$
\mathcal{B}_{n}^{(\alpha)}(x ; \lambda)=\sum_{r=0}^{n} \frac{n !}{r !}\left[\sum_{k=0}^{[(n-r) / m]}(-1)^{k} \frac{h^{k}}{k !(n-r-m k) !} \mathcal{B}_{n-r-m k}^{(\alpha)}(\lambda)\right] g_{r}^{m}(x, h),
$$

which is just Eq. (3.3) of [23].

By setting $u=1$ and $v=0$ in Theorem 3.9 , we have the following corollary.

\section{Corollary 3.11}

$$
\mathcal{E}_{n}^{(\alpha)}(x ; \lambda)=\sum_{r=0}^{n} \frac{n !}{r !}\left[\sum_{k=0}^{[(n-r) / m]}(-1)^{k} \frac{h^{k}}{k !(n-r-m k) !} \mathcal{E}_{n-r-m k}^{(\alpha)}(\lambda)\right] g_{r}^{m}(x, h) .
$$

By setting $u=1$ and $v=1$ in Theorem 3.9, we have the following corollary.

\section{Corollary 3.12}

$$
\mathcal{G}_{n}^{(\alpha)}(x ; \lambda)=\sum_{r=0}^{n} \frac{n !}{r !}\left[\sum_{k=0}^{[(n-r) / m]}(-1)^{k} \frac{h^{k}}{k !(n-r-m k) !} \mathcal{G}_{n-r-m k}^{(\alpha)}(\lambda)\right] g_{r}^{m}(x, h) .
$$

When $v \alpha=1$, applying Lemma 1.7 to $P_{n}(x)=\mathcal{E}_{n}^{(\alpha-1)}(x ; \lambda)$ and $Q_{n}(x)=\mathcal{F}_{n}^{(\alpha)}(x ; \lambda ; u, v)$, we have the following theorem. 
Theorem 3.13 If $v \alpha=1$, then we have

$$
\mathcal{F}_{n}^{(\alpha)}(x ; \lambda ; u, v)=\sum_{m=0}^{n}\left(\begin{array}{l}
n \\
m
\end{array}\right) 2^{(u-1) \alpha} \mathcal{G}_{n-m}(\lambda) \mathcal{E}_{m}^{(\alpha-1)}(x ; \lambda)
$$

By setting $\lambda:=-\lambda, u=0$ and $v=1$ in Theorem 3.13 , and then multiplying $(-1)^{\alpha}$ on both sides of the result, we have the following corollary.

\section{Corollary 3.14}

$$
\mathcal{B}_{n}(x ; \lambda)=-\frac{1}{2} \sum_{m=0}^{n}\left(\begin{array}{l}
n \\
m
\end{array}\right) \mathcal{G}_{n-m}(-\lambda) x^{m} .
$$

By setting $u=1$ and $v=1$ in Theorem 3.13, we have the following corollary.

\section{Corollary 3.15}

$$
\mathcal{G}_{n}(x ; \lambda)=-\frac{1}{2} \sum_{m=0}^{n}\left(\begin{array}{l}
n \\
m
\end{array}\right) \mathcal{G}_{n-m}(\lambda) x^{m}
$$

which is just the case of $\alpha=1$ in (3.4).

When $v=1$ or $\alpha=0$, applying Lemma 1.7 to $P_{n}(x)=\mathcal{G}_{n}^{(\alpha-1)}(x ; \lambda)$ and $Q_{n}(x)=\mathcal{F}_{n}^{(\alpha)}(x ; \lambda$; $u, v)$, we can obtain the following theorem.

Theorem 3.16 If $v=1$ or $\alpha=0$, we have

$$
\mathcal{F}_{n}^{(\alpha)}(x ; \lambda ; u, v)=\sum_{m=0}^{n}\left(\begin{array}{l}
n \\
m
\end{array}\right) 2^{(u-1) \alpha} \mathcal{G}_{n-m}(\lambda) \mathcal{G}_{m}^{(\alpha-1)}(x ; \lambda) .
$$

By setting $\lambda:=-\lambda, u=0$ and $v=1$ in Theorem 3.13, and then multiplying $(-1)^{\alpha}$ on both sides of the result, we have the following corollary.

\section{Corollary 3.17}

$$
\mathcal{B}_{n}^{(\alpha)}(x ; \lambda)=\sum_{m=0}^{n}\left(\begin{array}{l}
n \\
m
\end{array}\right)\left(-\frac{1}{2}\right)^{\alpha} \mathcal{G}_{n-m}(-\lambda) \mathcal{G}_{m}^{(\alpha-1)}(x ;-\lambda) .
$$

When $\alpha=1$ in (3.17), it is just (3.15).

By setting $u=1$ and $v=1$ in Theorem 3.16, we have the following corollary.

\section{Corollary 3.18}

$$
\mathcal{G}_{n}^{(\alpha)}(x ; \lambda)=\sum_{m=0}^{n}\left(\begin{array}{l}
n \\
m
\end{array}\right) \mathcal{G}_{n-m}(\lambda) \mathcal{G}_{m}^{(\alpha-1)}(x ; \lambda),
$$

which is equal to (3.8). 
If $\alpha=0$ in Theorem 3.16, we have:

\section{Corollary 3.19}

$$
x^{n}=\sum_{m=0}^{n}\left(\begin{array}{l}
n \\
m
\end{array}\right) \mathcal{G}_{n-m}(\lambda) \mathcal{G}_{m}^{(-1)}(x ; \lambda)
$$

\section{Hermite-based generalized Apostol type polynomials}

Finally, we give a generation of the generalized Apostol type polynomials.

The two-variable Hermite-Kampé de Fériet polynomials (2VHKdFP) $H_{n}(x, y)$ are defined by the series [31]

$$
H_{n}(x, y)=n ! \sum_{r=0}^{[n / 2]} \frac{x^{n-2 r} y^{r}}{r !(n-2 r) !}
$$

with the following generating function:

$$
\exp \left(x t+y t^{2}\right)=\sum_{n=0}^{\infty} \frac{t^{n}}{n !} H_{n}(x, y)
$$

And the 2VHKdFP $H_{n}(x, y)$ are also defined through the operational identity

$$
\exp \left(y \frac{\partial^{2}}{\partial x^{2}}\right)\left\{x^{n}\right\}=H_{n}(x, y)
$$

Acting the operator $\exp \left(y \frac{\partial^{2}}{\partial x^{2}}\right)$ on (1.14), and by the identity [32]

$$
\exp \left(y \frac{\partial^{2}}{\partial x^{2}}\right)\left\{\exp \left(-a x^{2}+b x\right)\right\}=\frac{1}{\sqrt{1+4 a y}} \exp \left(-\frac{a x^{2}-b x-b^{2} y}{1+4 a y}\right)
$$

we define the Hermite-based generalized Apostol type polynomials ${ }_{H} \mathcal{F}_{n}^{(\alpha)}(x, y ; \lambda ; u, v)$ by the generating function

$$
\left(\frac{2^{u} z^{v}}{\lambda e^{t}+1}\right)^{\alpha} \cdot e^{x t+y t^{2}}=\sum_{n=0}^{\infty}{ }_{H} \mathcal{F}_{n}^{(\alpha)}(x, y ; \lambda ; u, v) \frac{t^{n}}{n !} \quad(|t|<|\log (-\lambda)|)
$$

Clearly, we have

$$
{ }_{H} \mathcal{F}_{n}(x, y ; \lambda ; u, v)={ }_{H} \mathcal{F}_{n}^{(1)}(x, y ; \lambda ; u, v) .
$$

From the generating function (4.5), we easily obtain

$$
\frac{\partial}{\partial x}{ }_{H} \mathcal{F}_{n}^{(\alpha)}(x, y ; \lambda ; u, v)=n_{H} \mathcal{F}_{n-1}^{(\alpha)}(x, y ; \lambda ; u, v)
$$

and

$$
\frac{\partial}{\partial y}{ }_{H} \mathcal{F}_{n}^{(\alpha)}(x, y ; \lambda ; u, v)=n(n-1)_{H} \mathcal{F}_{n-2}^{(\alpha)}(x, y ; \lambda ; u, v),
$$


which can be combined to get the identity

$$
\frac{\partial^{2}}{\partial x^{2}}{ }_{H} \mathcal{F}_{n}^{(\alpha)}(x, y ; \lambda ; u, v)=\frac{\partial}{\partial y}{ }_{H} \mathcal{F}_{n}^{(\alpha)}(x, y ; \lambda ; u, v)
$$

Acting with the operator exp $y \frac{\partial^{2}}{\partial x^{2}}$ on both sides of (3.1), (3.5), (3.13), (3.18), and by using (4.3), we obtain

$$
\begin{aligned}
& { }_{H} \mathcal{F}_{n}^{(\alpha)}(x ; \lambda ; u, v)=\sum_{m=0}^{n}\left(\begin{array}{c}
n \\
m
\end{array}\right) \mathcal{F}_{n-m}^{(\alpha)}(\lambda ; u, v) H_{m}(x, y), \\
& { }_{H} \mathcal{F}_{n}^{(\alpha)}(x ; \lambda ; u, v)=\sum_{m=0}^{n}\left(\begin{array}{c}
n \\
m
\end{array}\right) \mathcal{F}_{n-m}^{(\alpha-1)}(\lambda ; u, v)_{H} \mathcal{F}_{m}(x ; \lambda ; u, v), \\
& { }_{H} \mathcal{F}_{n}^{(\alpha)}(x ; \lambda ; u, v)=\sum_{m=0}^{n}\left(\begin{array}{c}
n \\
m
\end{array}\right) 2^{(u-1) \alpha} \mathcal{G}_{n-m}(\lambda)_{H} \mathcal{E}_{m}^{(\alpha-1)}(x ; \lambda), \quad \text { where } v \alpha=1, \\
& { }_{H} \mathcal{G}_{n}^{(\alpha)}(x ; \lambda)=\sum_{m=0}^{n}\left(\begin{array}{c}
n \\
m
\end{array}\right) \mathcal{G}_{n-m}(\lambda)_{H} \mathcal{G}_{m}^{(\alpha-1)}(x ; \lambda), \quad \text { where } v=1 \text { or } \alpha=0,
\end{aligned}
$$

where ${ }_{H} \mathcal{E}_{n}^{(\alpha)}(x ; \lambda)$ and ${ }_{H} \mathcal{G}_{n}^{(\alpha)}(x ; \lambda)$ are the Hermite-based generalized Apostol-Euler polynomials and the Hermite-based generalized Apostol-Genocchi polynomials respectively, defined by the following generating functions:

$$
\begin{aligned}
& \left(\frac{2}{\lambda e^{t}+1}\right)^{\alpha} \cdot e^{x t+y t^{2}}=\sum_{n=0}^{\infty}{ }_{H} \mathcal{E}_{n}^{(\alpha)}(x ; \lambda) \frac{t^{n}}{n !} \quad(|t|<|\log (-\lambda)|), \\
& \left(\frac{2 t}{\lambda e^{t}+1}\right)^{\alpha} \cdot e^{x t+y t^{2}}=\sum_{n=0}^{\infty}{ }_{H} \mathcal{G}_{n}^{(\alpha)}(x ; \lambda) \frac{t^{n}}{n !} \quad(|t|<|\log (-\lambda)|) .
\end{aligned}
$$

\section{Competing interests}

The authors declare that they have no competing interests.

\section{Authors' contributions}

All authors contributed equally in writing this paper, and read and approved the final manuscript.

\section{Author details}

'Department of Mathematics, Yangzhou University, Yangzhou, Jiangsu 225002, People's Republic of China. ${ }^{2}$ Department of Mathematics, Chongqing Normal University, Chongqing Higher Education Mega Center, Huxi Campus, Chongqing, 401331, People's Republic of China.

\section{Acknowledgements}

Dedicated to Professor Hari M Srivastava.

The present investigation was supported, in part, by the National Natural Science Foundation of China under Grant 11226281, Fund of Science and Innovation of Yangzhou University, China under Grant 2012CXJ005, Research Project of Science and Technology of Chongaing Education Commission, China under Grant KJ120625 and Fund of Chongqing Normal University, China under Grant 10XLR017 and 2011XLZ07.

\section{Received: 10 December 2012 Accepted: 19 March 2013 Published: 28 March 2013}

\section{References}

1. Sándor, J, Crstici, B: Handbook of Number Theory, vol. II. Kluwer Academic, Dordrecht (2004)

2. Srivastava, HM, Choi, J: Series Associated with the Zeta and Related Functions. Kluwer Academic, Dordrecht (2001)

3. Srivastava, HM, Pintér, Á: Remarks on some relationships between the Bernoulli and Euler polynomials. Appl. Math. Lett. 17, 375-380 (2004)

4. Apostol, TM: On the Lerch zeta function. Pac. J. Math. 1, 161-167 (1951) 
5. Srivastava, HM: Some formulas for the Bernoulli and Euler polynomials at rational arguments. Math. Proc. Camb. Philos. Soc. 129, 77-84 (2000)

6. Luo, Q-M, Srivastava, HM: Some generalizations of the Apostol-Bernoulli and Apostol-Euler polynomials. J. Math. Anal. Appl. 308, 290-320 (2005)

7. Luo, Q-M: Apostol-Euler polynomials of higher order and Gaussian hypergeometric functions. Taiwan. J. Math. 10 917-925 (2006)

8. Horadam, AF: Genocchi polynomials. In: Proceedings of the Fourth International Conference on Fibonacci Numbers and Their Applications, pp. 145-166. Kluwer Academic, Dordrecht (1991)

9. Horadam, AF: Negative order Genocchi polynomials. Fibonacci Q. 30, 21-34 (1992)

10. Horadam, AF: Generation of Genocchi polynomials of first order by recurrence relations. Fibonacci Q. 30, 239-243 (1992)

11. Jang, L-C, Kim, T: On the distribution of the $q$-Euler polynomials and the $q$-Genocchi polynomials of higher order. J. Inequal. Appl. 2008, 1-9 (2008)

12. Luo, Q-M: Fourier expansions and integral representations for the Genocchi polynomials. J. Integer Seq. 12, 1-9 (2009)

13. Luo, Q-M: q-extensions for the Apostol-Genocchi polynomials. Gen. Math. 17, 113-125 (2009)

14. Luo, Q-M: Extension for the Genocchi polynomials and its Fourier expansions and integral representations. Osaka J. Math. 48, 291-309 (2011)

15. Luo, Q-M, Srivastava, HM: Some generalizations of the Apostol-Genocchi polynomials and the Stirling numbers of the second kind. Appl. Math. Comput. 217, 5702-5728 (2011)

16. Dattoli, G, Srivastava, HM, Zhukovsky, K: Orthogonality properties of the Hermite and related polynomials. J. Comput. Appl. Math. 182, 165-172 (2005)

17. Appell, P: Sur une classe de polynomials. Ann. Sci. Éc. Norm. Super. 9, 119-144 (1880)

18. He, MX, Ricci, PE: Differential equation of Appell polynomials via the factorization method. J. Comput. Appl. Math. 139, 231-237 (2002)

19. Ben Cheikh, Y, Chaggara, H: Connection problems via lowering operators. J. Comput. Appl. Math. 178, 45-61 (2005)

20. Garg, M, Jain, K, Srivastava, HM: Some relationships between the generalized Apostol-Bernoulli polynomials and Hurwitz-Lerch zeta functions. Integral Transforms Spec. Funct. 17, 803-815 (2006)

21. Lin, S-D, Srivastava, HM, Wang, P-Y: Some expansion formulas for a class of generalized Hurwitz-Lerch zeta functions. Integral Transforms Spec. Funct. 17, 817-827 (2006)

22. Liu, HM, Wang, WP: Some identities on the Bernoulli, Euler and Genocchi polynomials via power sums and alternate power sums. Discrete Math. 309, 3346-3363 (2009)

23. Lu, D-Q: Some properties of Bernoulli polynomials and their generalizations. Appl. Math. Lett. 24, 746-751 (2011)

24. Luo, Q-M: The multiplication formulas for the Apostol-Bernoulli and Apostol-Euler polynomials of higher order. Integral Transforms Spec. Funct. 20, 377-391 (2009)

25. Luo, Q-M, Srivastava, HM: Some relationships between the Apostol-Bernoulli and Apostol-Euler polynomials. Comput. Math. Appl. 51, 631-642 (2006)

26. Ozden, H, Simsek, Y, Srivastava, HM: A unified presentation of the generating functions of the generalized Bernoulli, Euler and Genocchi polynomials. Comput. Math. Appl. 60, 2779-2787 (2010)

27. Yang, S-L: An identity of symmetry for the Bernoulli polynomials. Discrete Math. 308, 550-554 (2008)

28. Yang, SL, Qiao, ZK: Some symmetry identities for the Euler polynomials. J. Math. Res. Expo. 30(3), 457-464 (2010)

29. Lu, D-Q, Srivastava, HM: Some series identities involving the generalized Apostol type and related polynomials. Comput. Math. Appl. 62, 3591-3602 (2011)

30. Gould, HW, Hopper, AT: Operational formulas connected with two generalizations of Hermite polynomials. Duke Math. J. 29, 51-63 (1962)

31. Appell, P, Kampé de Fériet, J: Functions hypergéométriques et hypersphériques: Polynómes d'Hermite. Gauthier-Villars, Paris (1926)

32. Dattoli, G, Khan, S, Ricci, PE: On Crofton-Glaisher type relations and derivation of generating functions for Hermite polynomials including the multi-index case. Integral Transforms Spec. Funct. 19, 1-9 (2008)

33. Dere, R, Simsek, Y: Normalized polynomials and their multiplication formulas. Adv. Differ. Equ. 2013, 31 (2013). doi:10.1186/1687-1847-2013-31

34. Dere, R, Simsek, Y: Genocchi polynomials associated with the umbral algebra. Appl. Math. Comput. 218, 756-761 (2011)

35. Dere, R, Simsek, Y: Applications of umbral algebra to some special polynomials. Adv. Stud. Contemp. Math. 22 433-438 (2012)

36. Kurt, B, Simsek, Y: Frobenius-Euler type polynomials related to Hermite-Bernoulli polynomials, analysis and applied math. AIP Conf. Proc. 1389, 385-388 (2011)

37. Srivastava, HM: Some generalizations and basic (or q-) extensions of the Bernoulli, Euler and Genocchi polynomials. Appl. Math. Inf. Sci. 5, 390-444 (2011)

38. Srivastava, HM, Kurt, B, Simsek, Y: Some families of Genocchi type polynomials and their interpolation functions. Integral Transforms Spec. Funct. 23, 919-938 (2012); see also corrigendum, Integral Transforms Spec. Funct. 23, 939-940 (2012)

39. Srivastava, HM, Ozarslan, MA, Kaanuglu, C: Some generalized Lagrange-based Apostol-Bernoulli, Apostol-Euler and Apostol-Genocchi polynomials. Russ. J. Math. Phys. 20, 110-120 (2013)

40. Srivastava, HM, Choi, J: Zeta and q-Zeta Functions and Associated Series and Integrals. Elsevier, Amsterdam (2012)

doi:10.1186/1687-2770-2013-64

Cite this article as: Lu and Luo: Some properties of the generalized Apostol-type polynomials. Boundary Value Problems 2013 2013:64 